\title{
A comparison of healthy human and swine articular cartilage dynamic indentation mechanics
}

\author{
S. Ronken - M. P. Arnold • H. Ardura García • \\ A. Jeger · A. U. Daniels • D. Wirz
}

Received: 11 April 2011 / Accepted: 2 July 2011 / Published online: 19 July 2011

(C) Springer-Verlag 2011

\begin{abstract}
Articular cartilage is a multicomponent, poroviscoelastic tissue with nonlinear mechanical properties vital to its function. A consequent goal of repair or replacement of injured cartilage is to achieve mechanical properties in the repair tissue similar to healthy native cartilage. Since fresh healthy human articular cartilage (HC) is not readily available, we tested whether swine cartilage (SC) could serve as a suitable substitute for mechanical comparisons. To a first approximation, cartilage tissue and surgical substitutes can be evaluated mechanically as viscoelastic materials. Stiffness measurements (dynamic modulus, loss angle) are vital to function and are also a non-destructive means of evaluation. Since viscoelastic material stiffness is strongly strain rate dependent, stiffness was tested under different loading conditions related to function. Stiffness of healthy $\mathrm{HC}$ and SC specimens was determined and compared using two nondestructive, mm-scale indentation test modes: fast impact and slow sinusoidal deformation. Deformation resistance (dynamic modulus) and energy handling (loss angle) were determined. For equivalent anatomic locations, there was no
\end{abstract}

S. Ronken $(\varangle) \cdot$ A. U. Daniels · D. Wirz

Laboratory of Biomechanics and Biocalorimetry (LOB2),

Biozentrum/Pharmazentrum,

Klingelbergstrasse 50-70, 4056 Basel, Switzerland

e-mail: sarah.ronken@gmail.com

M. P. Arnold · D. Wirz

Orthopedic Surgery and Skeletal Traumatology,

Kantonsspital Bruderholz, Bruderholz, Switzerland

H. Ardura García

Biomedical Engineering Section, Engineering Department, Universidad Iberoamericana, Mexico City, Mexico

A. Jeger

Institute for Sport and Sport Science, University of Basel Faculty

of Medicine, Basel, Switzerland difference in dynamic modulus. However, the $\mathrm{HC}$ loss angle was $\sim 35 \%$ lower in fast impact and $\sim 12 \%$ higher in slow sinusoidal mode. Differences seem attributable to age (young SC, older HC) but also to species anatomy and biology. Test mode-related differences in human-swine loss angle support use of multiple function-related test modes. Keeping loss angle differences in mind, swine specimens could serve as a standard of comparison for mechanical evaluation of e.g. engineered cartilage or synthetic repair materials.

Keywords Dynamic modulus - Energy dissipation . Human articular cartilage $\cdot$ Swine cartilage $\cdot$ Mechanical properties

\section{Introduction}

Articular cartilage is comprised mostly of extracellular material that is produced by a small number of chondrocytes. These cells have no direct blood supply and receive nutrition from synovial fluid and the subchondral bone plate (O'Hara et al. 1990). Cartilage must withstand millions of dynamic loads each year that are often multiples of body weight. It distributes intra-articular loads in a manner which serves to reduce localized stress concentrations in the underlying bone, and this may reduce the possibility of activity-related bone trauma. In the majority of individuals, articular cartilage is able to do this for decades after skeletal maturity without undergoing appreciable damage or wear itself.

One consequence of the above is that no synthetic implantable materials or structures are yet available which come close matching the mechanical properties and durability of articular cartilage. As a result, surgical repair of articular cartilage is reliant on either (a) joint replacement with metal, ceramic and polymer components, (b) autograft, allograft or xenograft transplantation, (c) medical and 
surgical treatments designed to rejuvenate cartilage or (d) surgical resurfacing of portions of the articular cartilage with engineered tissue (Fortin et al. 2002; Brown and Cruess 1982; Peterson et al. 2002; Hunziker 2002; Korhonen et al. 2002a). Assuming that repair products for damaged cartilage should mimic the biomechanical properties of healthy human cartilage, the best relative measure of their success is to determine to what extent the resultant structures have mechanical properties which resemble those of healthy human articular cartilage. But this poses another problem-such human tissue is not readily available for ex-vivo use as a reference material-i.e. to be subjected to the same mechanical tests as candidate synthetic structures, grafts, rejuvenated cartilage or engineered cartilage. In contrast, healthy animal articular cartilage is readily available for such purposes. In order to draw conclusions from animal models, one then has to ascertain the extent to which such animal cartilage has the same mechanical properties as human cartilage.

The response to non-damaging dynamic compressive loads has two aspects. The first is stiffness, or the amount of deformation in response to load. If cartilage stiffness is too low, it can become so thin under load that the load is effectively transmitted directly to the underlying bone. If cartilage stiffness is too high, a load is likely to become focused in a small region and can cause pain or tissue damage. The second response is energy handling, or the extent to which energy imparted by deformation is either stored or dissipated. If cartilage stores energy, it rapidly springs back to shape when a load is removed. If it instead dissipates some of the deformation energy (as heat), it returns to shape more slowly, and the peak loads in the cartilage itself and in underlying bone are reduced. This is a means of damage protection.

Cartilage is a complex "material" because it behaves in some ways like a sponge (porous), like a spring (elastic) and like a liquid (viscous). Thus, cartilage can be described as a "poroviscoelastic solid." The stiffness and energy handling of such complex materials depend on the rate at which they are deformed. As a result, there are special definitions for stiffness $-E^{*}=$ dynamic modulus, and energy handling $\delta=$ loss angle, methods for measuring them and reasons for doing so under different conditions which mimic cartilage dynamic mechanical function. The correlation between the storage modulus $\left(E^{\prime}\right)$, the loss modulus $\left(E^{\prime \prime}\right)$, the dynamic modulus $\left(E^{*}\right)$ and the loss angle $(\delta)$ for a viscoelastic material is shown in Fig. 1. This shows that at least two of those parameters are needed in order to describe a viscoelastic material properly. In previous studies, the focus was mainly on determining the stiffness (Schinagl et al. 1997; Kleemann et al. 2005; Lyyra et al. 1999; Stolz et al. 2004; Franke et al. 2007; Niederauer et al. 2004), and only a few studies also determined which part of the energy was dissipated (Park et al. 2004; Oakley et al. 2004; Appleyard 2003). Determining the mechanical properties of cartilage is typically done in

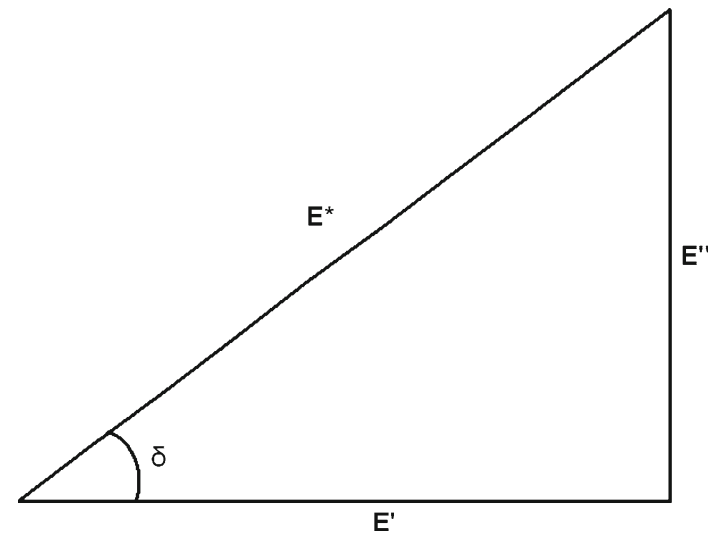

Fig. 1 Schematic representation of the correlation between the dynamic modulus $E^{*}$, the storage modulus $E^{\prime}$, the loss modulus $E^{\prime \prime}$ and the loss angle $\delta$ in a viscoelastic material

confined compression (Jurvelin et al. 1997; Korhonen et al. 2002b; Schinagl et al. 1997), unconfined compression (Park et al. 2004; Jurvelin et al. 1997; Korhonen et al. 2002b; Ficklin et al. 2007; Kleemann et al. 2005) or indentation (Lyyra et al. 1999; Oakley et al. 2004; Nugent et al. 2004; Mow et al. 1989; Korhonen et al. 2002b; Jurvelin et al. 1990; Appleyard 2003). Most of these studies determine the stiffness of the cartilage in slow compression, e.g. stress relaxation (Schinagl et al. 1997; Nieminen et al. 2004; Korhonen et al. 2002b; Jurvelin et al. 1997; Ficklin et al. 2007) or creep (Athanasiou et al. 1994; Mow et al. 1989; Jurvelin et al. 1990; Kleemann et al. 2005). However, besides undergoing slow quasi-cyclic deformations (e.g. while someone stands in place), cartilage also functions in sudden transient deformations which occur during gait. This is important, because as mentioned above dynamic stiffness parameters of poroviscoelastic materials like cartilage are extremely dependent on deformation rate.

The aims of this study were to (a) measure and compare $E^{*}$ and $\delta$ of cartilage using the authors' own protocols for testing in slow quasi-cyclic deformation and fast impact indentation and (b) to determine and compare results for healthy swine specimens and healthy human cartilage. The overall goal was to establish whether swine specimens can serve as a reference material for evaluating the extent to which articular cartilage replacement and repair strategies achieve cartilage-like mechanical behaviour.

\section{Materials and methods}

\subsection{Materials}

\subsubsection{Swine cartilage}

Swine cartilage (SC) was obtained from the knee of 10-month-old swine. Cylindrical osteochondral plugs of 7.6 
Fig. 2 Example of a swine (left) and a human (right) piece of cartilage. White bar is $1 \mathrm{~cm}$
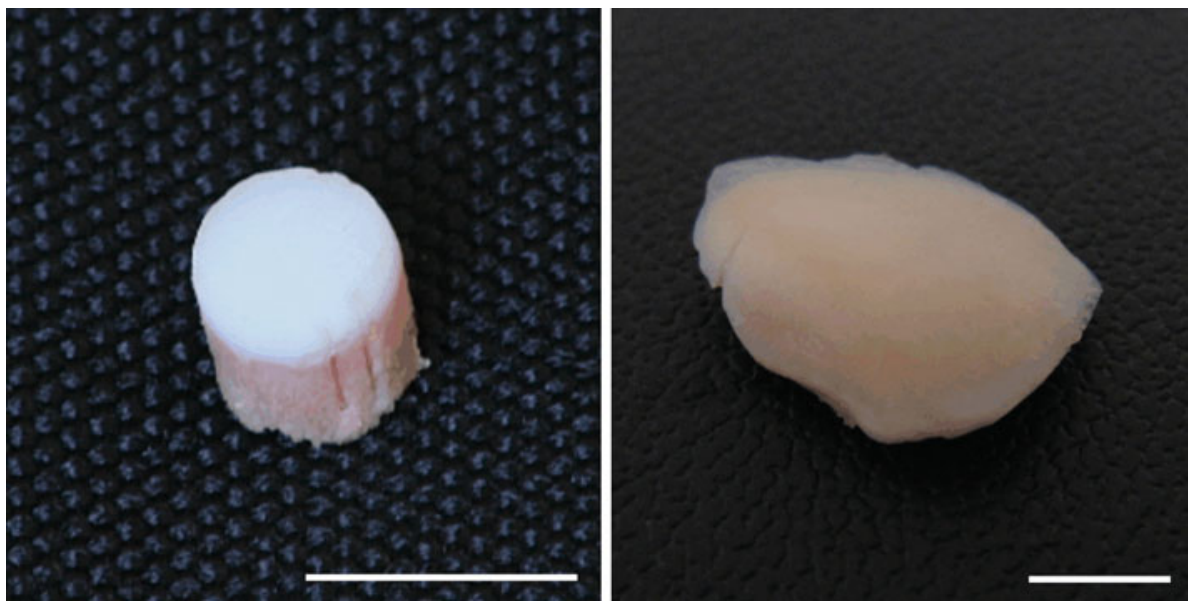

$\mathrm{mm}$ in diameter (Fig. 2; left) were harvested using a standard diamond core-drill designed for mosaicplasty (Synthes, Oberdorf, Switzerland). Plugs were harvested from the lateral patella (LP), the medial and lateral patellar groove (MPG \& LPG) and from the medial and lateral condyles (MC \& LC). The estimated thickness of the swine cartilage was in the range of 1.0-3.2 mm. The thickness of the LC was 1.1-2.3 $\mathrm{mm}$. The samples were frozen at $-24^{\circ} \mathrm{C}$ and hydrated with phosphate-buffered saline (PBS) to prevent drying. Prior to testing, the samples were thawed until room temperature and kept wet with PBS during testing.

\subsubsection{Human cartilage}

Human articular knee cartilage (HC) tissues of 1-2 cm in diameter were collected from full-thickness biopsies of the lateral femoral condyle of 8 fresh human cadavers ( 2 men and 6 women, median age: 57 years, range: 42-69) at the Department of pathology of the local University Hospital following informed consent by relatives and in accordance with the requirements of the Local Ethical Committee. Tissue was only harvested from knees without macroscopic signs of degenerative arthritis (Fig. 2; right). The estimated cartilage thickness was $1.2-2.7 \mathrm{~mm}$. The samples were frozen at $-24^{\circ} \mathrm{C}$ and hydrated with PBS for later use. Prior to testing, the samples were thawed in PBS at room temperature and kept wet during testing.

\subsection{Methods}

Two micro-indentation methods were used as previously described (Ardura Garcia et al. 2008; Wirz et al. 2008; Arnold et al. 2011) to determine the dynamic stiffness parameters (dynamic modulus $E^{*}$ and loss angle $\delta$ ) of cartilage, meniscus and possible implant materials. The dynamic modulus is a measure of the deformation resistance of a material. The loss angle is a measure of the energy dissipation. If a cyclic load is applied to a viscoelastic material, the strain will lag the stress (Lakes 1999).

The dynamic stiffness parameters of poroviscoelastic materials, i.e. cartilage, are extremely strain rate dependent. Besides that, cartilage functions in two different loading regimes - the sudden transient deformations which occur during gait, and the slow quasi-cyclic deformations which cause fluid to move in and out of cartilage and thus provide a means for nutrition. Thus, both a Fast Impact Mode and a Slow Sinusoidal Mode test method were developed. The dynamic modulus was calculated as described by Wirz et al. (2008) and Kren et al. (2005). The loss angle was calculated directly from the lag of the displacement and load curves.

\subsubsection{Fast impact (FI) mode}

To simulate the impact velocity in gait, a fast impact microindentation instrument was used. This is a modified version of an instrument developed at the Minsk Institute of Physics (Kren et al. 2005). A pendulum-mounted spherical indenter (diameter: $1.0 \mathrm{~mm} ; 1.9 \mathrm{~g}$ ) falls down on the specimen under gravitational force. The motion of the indenter is captured electromagnetically during indentation and rebound. On each specimen, 10 replicate measurements were performed on the same spot at $\sim 20 \mathrm{~s}$ time intervals. Resultant $E^{*}$ and $\delta$ were calculated for each impact and then each set was averaged to get one set of specimen values.

\subsubsection{Slow sinusoidal (SS) mode}

To simulate nutrition in cartilage specimens, a Synergie 100 MTS ${ }^{\circledR}$ mechanical testing instrument was used to perform slow sinusoidal micro-indentations. A spherical indenter (diameter $\sim 3.2 \mathrm{~mm}$ ) was moved sinusoidally under computer software control. The frequency was $0.1 \mathrm{~Hz}$, and the indentation depth was $\sim 0.05 \mathrm{~mm}$. The maximum speed was 
Fig. 3 Typical example of the force and displacement curves of a swine (line) and human (dotted line) sample in fast impact (left) and slow sinusoidal (right) mode
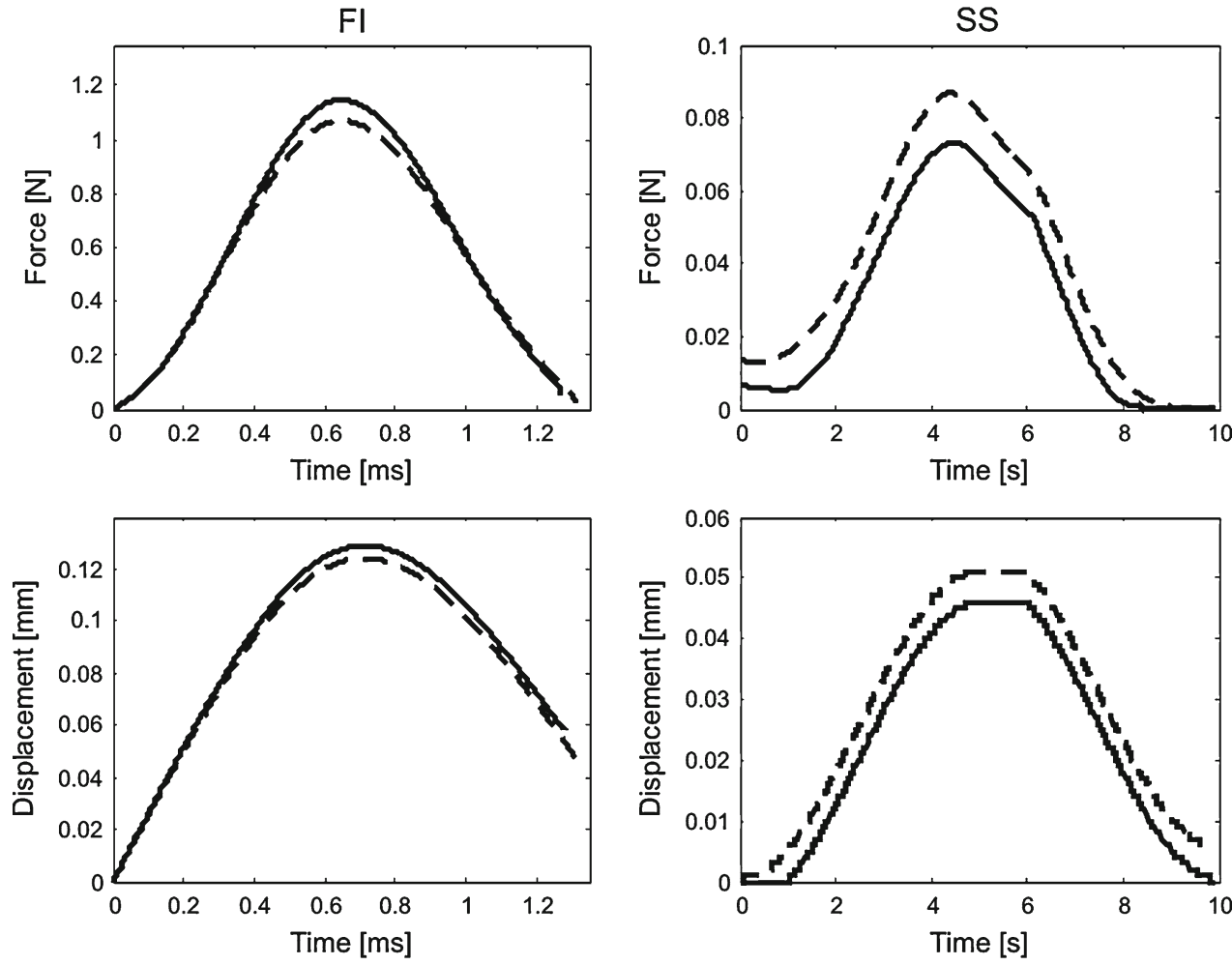

Swine $-\boldsymbol{-}$ Human $\sim 0.015 \mathrm{~m} / \mathrm{s}$. The same specimens were measured as in FI mode. On each specimen, 5 replicate measurements were performed at intervals of $\sim 2$ min on the same spot. Resultant $E^{*}$ and $\delta$ were averaged to get one set of specimen values.

\subsection{Statistical analysis}

A one-sided Wilcoxon Rank Sum test was performed on both the $E^{*}$ and $\delta$ data sets $(p<0.05)$. To quantify the spread in the data, the median absolute deviation was calculated and divided by the median to normalize and thus allow comparisons of the spreads within the data. Statistical analysis was accomplished using R (R Foundation for Statistical Computing, Austria).

\section{Results}

In FI mode, for both $\mathrm{HC}$ and $\mathrm{SC}$ specimens, both force and displacement versus time were smooth parabolic curves but with peak force and peak displacement not occurring at the same time (Fig. 3). This phase difference was attributable to the essentially viscoelastic behaviour of cartilage at high strain rates.
In SS mode, the applied displacement was sinusoidal as specified by the software driving displacement. There was again a difference in the time at which force and displacement reached a maximum, again attributable to viscoelasticity. In addition, however, for both $\mathrm{HC}$ and $\mathrm{SC}$ specimens, the resultant force-time curves were not sinusoidal.

The $E^{*}$ and $\delta$ calculated from the raw SC data are shown in Fig. 4. At any anatomic location, $E^{*}$ was significantly higher and $\delta$ was significantly lower in FI mode compared to SS mode. In cartilage and also most synthetic viscoelastic materials, faster deformation rates result in higher $E^{*}$ and lower $\delta$. Also, some significant differences in these parameters were found among the various locations on the swine knee for both testing modes.

The $E^{*}$ in FI mode on the LC and the MC was lower compared to the LP, the LPG and the MPG. In SS mode, fewer differences were found. The $E^{*}$ on the LC was lower than on the LPG and the MPG and also lower on the MC compared to the MPG.

The $\delta$ of the LC was lower compared to the LP, LPG and MPG and lower on the MC than on the LP in FI mode. In SS mode, there were no differences in $\delta$ among the various locations.

For both test modes, there was no statistically significant difference in $E^{*}$ between human and swine LC (Fig. 5). 
Fig. 4 Box-and-Whisker plot of the dynamic modulus (up) and the loss angle (bottom) of the swine specimens at fast impact (left) and slow sinusoidal (right) mode. $L C$ lateral condyle, $M C$ medial condyle, $L P$ lateral patella, $L P G$ lateral patellar groove, $M P G$ medial patellar groove. Horizontal lines indicate a significant difference with $p<0.05$
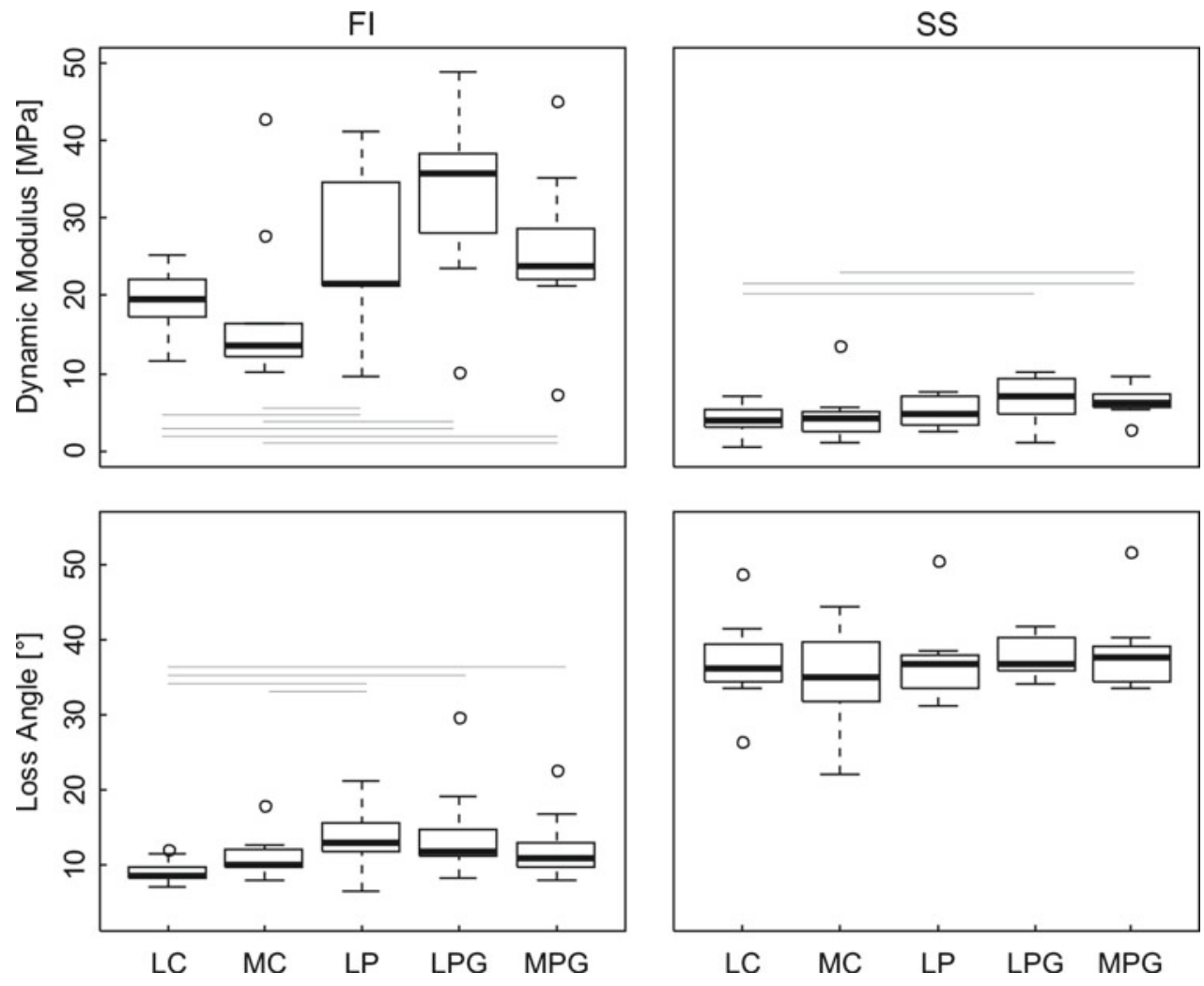
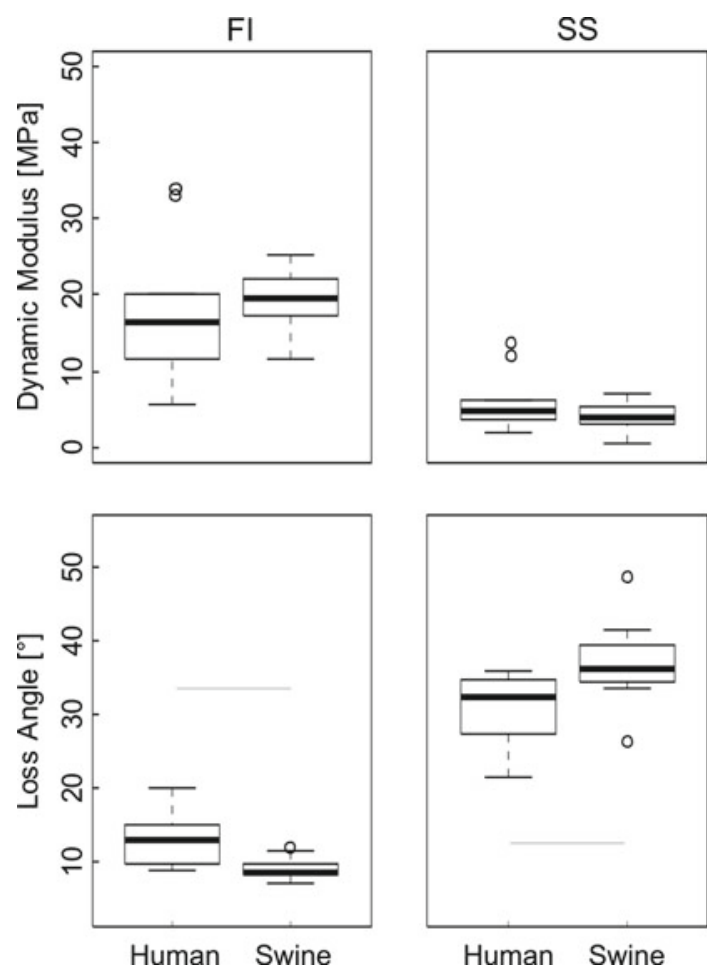

Fig. 5 Box-and-Whisker plot of the dynamic modulus (up) and the loss angle (bottom) of the human and swine lateral condyle $(L C)$ specimens at fast impact $(F I)$ and slow sinusoidal $(S S)$ mode. Horizontal lines indicate a significant difference with $p<0.05$
However, $\delta$ was significantly higher in FI mode and lower in SS mode for the HC compared to the SC.

The median absolute deviation was calculated and divided by the median to normalize and thus allow comparisons of the spreads within the data (Table 1 ). The normalized spread in $E^{*}$ of the $\mathrm{HC}$ specimens was $26 \%$ for both FI and SS mode. For the SC, the normalized spread was between 10 and 52\% depending on location and test mode. The overall normalized variability within the SC samples was $36 \%$ for FI mode and $34 \%$ for SS mode. The normalized spread was lower for the $\delta$ compared to the $E^{*}$. For the HC specimens, the normalized spread was $21 \%$ in FI mode and $8 \%$ in SS mode. In the SC specimens, the normalized spread was between $5 \%$ and $19 \%$. The overall normalized variation within the SC samples was $16 \%$ in FI mode and 7\% in SS mode. The normalized spread in $\delta$ was lower in SS mode compared to FI mode.

\section{Discussion}

\subsection{Effect of specimen dimensions}

To ensure that tissue specimen thickness would not influence results, indentation in both modes was limited to less than $\sim 10 \%$ of tissue thickness (Bueckle 1973). Further, Niederauer et al. (2004) showed that cartilage stiffness is not correlated with cartilage thickness. We also used a small 
Table 1 Median absolute deviation divided by the median from the human and swine specimens on the various locations

\begin{tabular}{|c|c|c|c|c|c|c|c|c|}
\hline \multirow[t]{2}{*}{ Parameter } & \multirow[t]{2}{*}{ Mode } & \multirow{2}{*}{$\begin{array}{l}\text { Human } \\
\text { LC }(\%)\end{array}$} & \multicolumn{6}{|l|}{ Swine } \\
\hline & & & LC $(\%)$ & $\operatorname{MC}(\%)$ & LP $(\%)$ & LPG (\%) & MPG $(\%)$ & All (\%) \\
\hline \multirow[t]{2}{*}{$E^{*}$} & FI & 26 & 14 & 21 & 52 & 16 & 10 & 36 \\
\hline & SS & 26 & 24 & 31 & 43 & 33 & 14 & 34 \\
\hline \multirow[t]{2}{*}{$\delta$} & FI & 21 & 11 & 19 & 18 & 8 & 19 & 16 \\
\hline & SS & 8 & 6 & 12 & 6 & 5 & 6 & 7 \\
\hline
\end{tabular}

$L C$ lateral condyle, $M C$ medial condyle, $L P$ lateral patella, $L P G$ lateral patellar groove, $M P G$ medial patellar groove

indenter diameter compared to the diameter of the osteochondral plugs, in order to minimize specimen dimension effects in directions parallel to the specimen articular surface.

\subsection{Effects of test mode on force-time}

and displacement-time data

As expected, since cartilage is partly a viscoelastic material, the maxima in force-time and displacement-time data (Fig. 3) occurred at different times in both FI and SS modes (Lakes 1999). This phase shift is due to energy losses resulting from internal friction. In FI mode, the high strain rate coupled with the low permeability of water through cartilage resulted in essentially viscoelastic rather than more complex poroviscoelastic behaviour, and thus, smooth changes in both force and displacement with time were observed. In contrast in SS mode, the strain rate is low, and at $0.1 \mathrm{~Hz}$ there is time for water to move through the structure in response to load in spite of the low permeability. The response of cartilage is then poroviscoelastic. In SS mode-and thus under poroviscoelastic response conditions and at smaller displacements than in FI mode-discontinuities were observed in both the displacement-time and force-time curves. The discontinuities in the displacement-time curves were due to measurement artefacts. These were uniform throughout the measurements. The measurement system was unable to resolve the small changes in displacement with time occurring near the peak of the applied sinusoidal wave form. In contrast, the non-sinusoidal variations in the force-time curves were seen only with cartilage specimens and not seen with essentially elastic synthetic materials (data not shown), indicating that this was not an artefact. For cartilage, near the peak of displacement, the change in the indentation depth with time was extremely small. Therefore, the non-sinusoidal decline in force during this time can likely be attributed to energy loss of the cartilage.

\subsection{Effects of cartilage test mode and anatomic location on $E^{*}$ and $\delta$}

As expected, $E^{*}$ was higher and $\delta$ was lower at all swine anatomic locations in FI mode compared to SS mode (Fig. 4).
This is the normal response of materials exhibiting some viscoelastic behaviour when tested at a high strain rate (FI) versus a low strain rate (SS) (Park et al. 2004).

There were also marked differences in $E^{*}$ as a function of anatomic location in both FI and SS modes. However, there were more significant differences in $E^{*}$ as a function of anatomic location for FI mode than for SS mode, and only FI mode detected any significant differences in $\delta$ as a function of anatomic location. Thus, FI mode seems more sensitive detecting variations in cartilage stiffness parameters between different locations. This is somewhat unexpected since as described above, the high strain rate and short duration of the FI mode limit the motion of water through cartilage. As a result, one might expect that the FI method would be less sensitive to changes in the proteoglycan portion of the cartilage structure and thus less sensitive overall. This was not the case, and possible reasons for this are discussed later below.

In any case, the differences in test mode sensitivity to anatomic location point up the importance of evaluating cartilage stiffness parameters at substantially different strain rates that are related to the spectrum of strain rates that occur in vivo. Also, as discussed later, there are other phenomena which are only revealed by using more than one strain rate.

It is already known that the stiffness of cartilage varies at different locations in human (Lyyra et al. 1999) and sheep (Appleyard 2003) knees. Lyyra et al. (1999) found the highest stiffness in the load-bearing areas of the condyles, where we found the highest $E^{*}$ in the lateral patellar groove and the lowest $E^{*}$ on the lateral and the medial condyles. This difference might be due to a different loading pattern in the swine knee compared to the human knee.

To quantify the normalized spread within the different specimens, the median absolute deviation was divided by the median. The results showed a large spread between the various samples (Table 1 ). The normalized spread in $E^{*}$ between the HC samples was 26\%. Lyyra et al. (1999) determined the coefficient of variation which was $29 \%$ between individuals. The $E^{*}$ of the HC specimens did not correlate with age in this study. The spread might be due to the cartilage being exposed to another load pattern, e.g. sports, body weight. Another reason could be differences in cartilage thickness, 
although thickness and stiffness were not shown to be correlated in the study of Niederauer et al. (2004).

The normalized spread between subjects and between the different locations was smaller in $\delta$ than in $E^{*}$ (Table 1$)$. The spread was the smallest in the $\delta$ in the SS mode. In the early stage of osteoarthritis, more proteoglycans are produced by the chondrocytes (Cs-Szabó et al. 1997), which changes the capability of the cartilage to hold the water. In addition to the increase in proteoglycans during osteoarthritis, cartilage collagen declines (Lippiello et al. 1977; Pullig et al. 1999) and damage occurs in the existing collagen in the superficial and upper mid zone (Hollander et al. 1995). Due to the smaller variation in $\delta$ and the changes occurring in osteoarthritic cartilage, osteoarthritis might be easier to detect by looking at the $\delta$ instead of the $E^{*}$. Stolz et al. (2009) showed that detection of osteoarthritis is possible in an early state using AFM measurements; however, they only determined $E^{*}$. The difference between healthy and osteoarthritic cartilage might be even clearer if the loss angle is also determined.

\subsection{Differences in $E^{*}$ and $\delta$ between human and swine LC specimens}

The $E^{*}$ of the $\mathrm{HC}$ and $\mathrm{SC}$ was not significantly different in either FI or SS mode. This was encouraging as a purpose of this study was to evaluate whether healthy SC can serve as a stand-in for healthy $\mathrm{HC}$ in stiffness parameter tests. However, the trends were that $\mathrm{SC}$ was stiffer than $\mathrm{HC}$ in FI mode but less stiff in SS mode. In contrast to the $E^{*}$ results, the $\delta$ of SC was significantly $(\sim 35 \%)$ lower in FI mode and $(\sim 12 \%)$ higher in SS mode compared to HC. Overall, the swine versus human differences were not enormous, and that is encouraging. However, the results do raise two questions. First, why were the results somewhat different for $\mathrm{HC}$ and $\mathrm{SC}$, and second why were the swine versus human relationships opposite for FI mode compared to SS mode?

The relatively small swine versus human differences in a given test (FI or SS) seem likely to be due to a difference in composition of the cartilage. Composition differences could be expected for two reasons. First, the swine specimens were from young mature, healthy animals (age $\sim 10$ months) while the human specimens - although visually judged to be healthy-were from humans far past skeletal maturity (age 42-69 years). A limitation of this study is that, since older human and younger swine cartilage were measured, it was not possible to distinguish whether differences found between $\mathrm{HC}$ and SC were due to age or species structural variations or some combination of the two. In addition, the swine data for various anatomic locations show considerable variation, no doubt reflecting load-related needs. In this light, it may be that differences in the stance and gait of swine versus humans result in different load-related needs on the LC and thus differences in structure and mechanical properties between swine and human LC cartilage.

The second question remains: why were the swine versus human relationships for LC cartilage in both $E^{*}$ (trend) and $\delta$ (significant) opposite for FI mode compared to SS mode? These opposite results suggest the following.

In FI mode, there is little or no movement of water and thus the $E^{*}$ values may mostly reflect mechanics of crosslinked collagen. Cross-linking of collagen or other polymers (e.g. latex) generally causes them to behave more like rubber-less viscously and more elastically (higher $E^{*}$ lower $\delta)$. Thus in FI mode, the lower $E^{*}$ and higher $\delta$ for $\mathrm{HC}$ compared to SC might have been attributable to a relatively lower amount of cross-linking of collagen in the (older) $\mathrm{HC}$ than in the (younger) SC. However, it is reported that collagen crosslinking is likely to increase with age (Loeser 2004). Also, it has been shown that the collagen fibre arrangement is similar in HC and SC (Kääb et al. 1998). Consequently, the difference found is perhaps attributable to a species difference in the amount of collagen cross-linking.

Conversely, in SS mode, $\mathrm{HC}$ exhibited a higher $E^{*}$ (trend) and lower $\delta$ (significant) compared to SC. As described above, a great part of the cartilage mechanical response during slow deformation $(0.1 \mathrm{~Hz}$ in this case $)$ is due to the nature of the motion of water in the proteoglycan portion of the cartilage structure. A relatively lower amount of water in the proteoglycans could be expected to result in less viscous behaviour-i.e. higher $E^{*}$ and lower $\delta$-as seen here for $\mathrm{HC}$ (older) specimens versus SC (younger) cartilage specimens. Indeed, a decline in proteoglycan water content of cartilage with age has been reported (Wells et al. 2003).

The above-described contrasting, structurally explainable results for $E^{*}$ and $\delta$ for the FI versus the SS test modes support the idea that cartilage mechanics should be evaluated over a range of representative load deformation conditions. Otherwise, the picture is incomplete, and misleading conclusions could be drawn. Results here are for two extremes. They suggest that even more information related to age and disease induced variations in cartilage structure might be revealed by a more thorough exploration of the effects of rate and amplitude of deformation. The results also point up that besides the modulus, the loss angle is an important dynamic stiffness parameter of cartilage and should therefore be a part of every test set that is meant to define the mechanical quality of cartilage or suitability of a cartilage repair material.

\section{Conclusions}

Swine cartilage dynamic modulus and loss angle varied significantly with anatomic location within the swine knee. More significant differences were seen in FI (fast impact) mode than in SS (slow sinusoidal) mode. In fact, in SS mode, 
no significant differences were seen in loss angle as a function of anatomic location. As discussed in detail above, it seems likely that in SS mode, water motion is the primary determinant of energy dissipation and does not change appreciably with anatomic location. This masks dissipation effects related to anatomic location differences in the collagen network. In FI mode, these differences become evident in the absence of water motion.

Differences in human-swine loss angle trends for FI (fast impact) versus SS (slow sinusoidal) test modes support the need for using multiple function-related test modes to more completely understand cartilage mechanical behaviour.

Finally, keeping loss angle differences in mind, swine specimens could serve as a standard of comparison for mechanical evaluation of e.g. surgically repaired cartilage, engineered cartilage or synthetic repair materials.

Acknowledgments H.-J. Wyss for the funds donated to University Basel and the Hardy \& Otto Frey-Zünd Stiftung Basel for the support.

\section{References}

Appleyard R (2003) Topographical analysis of the structural, biochemical and dynamic biomechanical properties of cartilage in an ovine model of osteoarthritis. Osteoarthr Cartil 11(1):65-77

Ardura Garcia H, Daniels AU, Wirz D (2008) Dual-mode dynamic functional stiffness of articular cartilage. Eur Cells Mater 16(1):7

Arnold MP, Daniels AU, Ronken S, García HA, Friederich NF, Kurokawa $\mathrm{T}$ et al (2011) Acrylamide polymer double-network hydrogels: candidate cartilage repair materials with cartilagelike dynamic stiffness and attractive surgery-related attachment mechanics. Cartilage: accepted for publication 1/2011

Athanasiou KA, Agarwal A, Dzida FJ (1994) Comparative study of the intrinsic mechanical properties of the human acetabular and femoral head cartilage. J Orthop Res 12(3):340-349

Brown KL, Cruess RL (1982) Bone and cartilage transplantation in orthopaedic surgery. A review. J Bone Joint Surg 64(2):270-279

Bueckle H (1973) Use of the hardness test to determine other material properties. In: Westbrook JW, Conrad H (eds) The science of hardness testing and its research applications. American Society for Metals, Materials Park pp 453-491

Cs-Szabó G, Melching LI, Roughley PJ, Glant TT (1997) Changes in messenger rna and protein levels of proteoglycans and link protein in human osteoarthritic cartilage samples. Arthr Rheum 40(6): 1037-1045

Ficklin T, Thomas G, Barthel JC, Asanbaeva A, Thonar EJ, Masuda $\mathrm{K}$ et al (2007) Articular cartilage mechanical and biochemical property relations before and after in vitro growth. J Biomech 40(16):3607-3614

Fortin PR, Penrod JR, Clarke AE, St-Pierre Y, Joseph L, Bélisle P et al (2002) Timing of total joint replacement affects clinical outcomes among patients with osteoarthritis of the hip or knee. Arthr Rheum 46(12):3327-3330

Franke O, Durst K, Maier V, Göken M, Birkholz T, Schneider H et al (2007) Mechanical properties of hyaline and repair cartilage studied by nanoindentation. Acta Biomater 3(6):873-881

Hollander AP, Pidoux I, Reiner A, Rorabeck C, Bourne R, Poole AR (1995) Damage to type II collagen in aging and osteoarthritis starts at the articular surface, originates around chondrocytes, and extends into the cartilage with progressive degeneration. J Clin Invest 96(6):2859-2869

Hunziker EB (2002) Articular cartilage repair: basic science and clinical progress. A review of the current status and prospects. Osteoarthr Cartil 10(6):432-463

Jurvelin J, Buschmann M, Hunziker E (1997) Optical and mechanical determination of poisson's ratio of adult bovine humeral articular cartilage. J Biomech 30(3):235-241

Jurvelin J, Kiviranta I, Säämänen A-M, Tammi M, Helminen HJ (1990) Indentation stiffness of young canine knee articular cartilage-influence of strenuous joint loading. J Biomech 23(12):1239-1246

Kääb MJ, Gwynn IA, Nötzli HP (1998) Collagen fibre arrangement in the tibial plateau articular cartilage of man and other mammalian species. J Anat 193(1):23-34

Kleemann RU, Krocker D, Cedraro A, Tuischer J, Duda GN (2005) Altered cartilage mechanics and histology in knee osteoarthritis: relation to clinical assessment (ICRS Grade). Osteoarthr Cartil 13(11):958-963

Korhonen RK et al (2002a) Comparison of the equilibrium response of articular cartilage in unconfined compression, confined compression and indentation. J Biomech 35(7):903-909

Korhonen RK, Laasanen MS, Töyräs J, Rieppo J, Hirvonen J, Helminen $\mathrm{HJ}$ et al (2002b) Importance of the superficial tissue layer for the indentation stiffness of articular cartilage. Med Eng Phys 24(2):99-108

Kren AP, Rudnitskii VA, Deikun IG (2005) Determining the viscoelastic parameters of vulcanisates by the dynamic indentation method using a non-linear deformation model. Int Polym Sci Tech 32(7):19-23

Lakes RS (1999) Viscoelastic solids. CRC Press, Boca Raton

Lippiello L, Hall D, Mankin HJ (1977) Collagen synthesis in normal and osteoarthritic human cartilage. J Clin Invest 59(4):593-600

Loeser RF (2004) Aging cartilage and osteoarthritis-what's the link? Science of aging knowledge environment : SAGE KE 2004(29): 31

Lyyra T, Kiviranta I, Väätäinen U, Helminen HJ, Jurvelin J (1999) In vivo characterization of indentation stiffness of articular cartilage in the normal human knee. J Biomed Mater Res 48(4):482-487

Mow V, Gibbs M, Lai W, Zhu W, Athanasiou KA (1989) Biphasic indentation of articular cartilage-II. A numerical algorithm and an experimental study. J Biomech 22(8/9):853-861

Niederauer GG, Niederauer GM, Cullen LC, Athanasiou KA, Thomas JB, Niederauer MQ (2004) Correlation of cartilage stiffness to thickness and level of degeneration using a handheld indentation probe. Ann Biomed Eng 32(3):352-359

Nieminen MT, Töyräs J, Laasanen MS, Silvennoinen J, Helminen HJ, Jurvelin J (2004) Prediction of biomechanical properties of articular cartilage with quantitative magnetic resonance imaging. J Biomech 37(3):321-328

Nugent GE, Law AW, Wong EG, Temple MM, Bae WC, Chen AC et al (2004) Site- and exercise-related variation in structure and function of cartilage from equine distal metacarpal condyle. Osteoarthr Cartil 12(10):826-833

Oakley SP, Lassere MN, Portek I, Szomor Z, Ghosh P, Kirkham BW et al (2004) Biomechanical, histologic and macroscopic assessment of articular cartilage in a sheep model of osteoarthritis. Osteoarthr Cartil 12(8):667-679

O’Hara BP, Urban JPG, Maroudas A (1990) Influence of cyclic loading articular cartilage. Ann Rheum Dis 49:536-539

Park S, Hung C, Ateshian G (2004) Mechanical response of bovine articular cartilage under dynamic unconfined compression loading at physiological stress levels. Osteoarthr Cartil 12:65-73

Peterson L, Brittberg M, Kiviranta I, Åkerlund EL, Lindahl A (2002) Autologous chondrocyte transplantation: biomechanics and long-term durability. Am J Sport Med 30(1):2-12 
Pullig O, Weseloh G, Swoboda B (1999) Expression of type VI collagen in normal and osteoarthritic human cartilage. Osteoarthr Cartil 7(2):191-202

Schinagl RM, Gurskis D, Chen AC, Sah RL (1997) Depth-dependent confined compression modulus of full-thickness bovine articular cartilage. J Orthop Res 15(4):499-506

Stolz M, Raiteri R, Daniels AU, VanLandingham MR, Baschong W, Aebi U (2004) Dynamic elastic modulus of porcine articular cartilage determined at two different levels of tissue organization by indentation-type atomic force microscopy. Biophys J 86(5): 3269-3283

Stolz M, Gottardi R, Raiteri R, Miot S, Martin I, Imer R et al (2009) Early detection of osteoarthritis and articular cartilage aging in mice and patient biopsies using atomic force microscopy. Nat Nanotechnol 4(3):186-192

Wells T, Davidson C, Mörgelin M, Bird JLE, Bayliss MT, Dudhia J (2003) Age-related changes in the composition, the molecular stoichiometry and the stability of proteoglycan aggregates extracted from human articular cartilage. Biochem J 370(1): 69-79

Wirz D, Kohler K, Keller B, Göpfert B, Hudetz D, Daniels AU (2008) Dynamic stiffness of articular cartilage by single impact micro-indentation (SIMI). J Biomech 41(Supplement 1):S172 